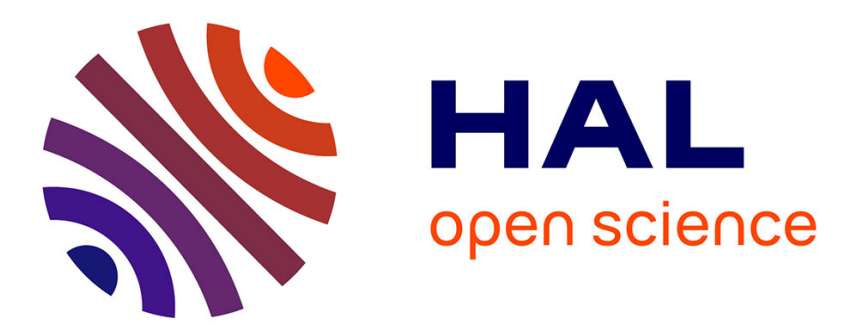

\title{
Étude des bandes d'absorption infrarouges v0-3, v0-4, v0-5 de l'acide bromhydrique gazeux à l'aide d'un spectromètre SISAM
}

\author{
P. Bernage, P. Niay, H. Bocquet, R. Houdart
}

\section{To cite this version:}

P. Bernage, P. Niay, H. Bocquet, R. Houdart. Étude des bandes d'absorption infrarouges v0-3, v0-4, v0-5 de l'acide bromhydrique gazeux à l'aide d'un spectromètre SISAM. Revue de Physique Appliquée, 1973, 8 (4), pp.333-335. 10.1051/rphysap:0197300804033300 . jpa-00243691

HAL Id: jpa-00243691

https://hal.science/jpa-00243691

Submitted on 1 Jan 1973

HAL is a multi-disciplinary open access archive for the deposit and dissemination of scientific research documents, whether they are published or not. The documents may come from teaching and research institutions in France or abroad, or from public or private research centers.
L'archive ouverte pluridisciplinaire HAL, est destinée au dépôt et à la diffusion de documents scientifiques de niveau recherche, publiés ou non, émanant des établissements d'enseignement et de recherche français ou étrangers, des laboratoires publics ou privés. 
Classification

Physics Abstracts

13.31

\title{
ÉTUDE DES BANDES D'ABSORPTION INFRAROUGES $v_{0-3}, v_{0-4}, v_{0-5}$ DE L'ACIDE BROMHYDRIQUE GAZEUX A L'AIDE D'UN SPECTROMÊTRE SISAM
}

\author{
P. BERNAGE, P. NIAY, H. BOCQUET et R. HOUDART \\ Laboratoire de Spectroscopie des molécules diatomiques $\left({ }^{*}\right)$, \\ UER de Physique Fondamentale, \\ Université des Sciences et Techniques de Lille, \\ 59650 Villeneuve D'Ascq, France
}

(Reçu le 18 juin 1973, révisé le 12 juillet 1973)

\begin{abstract}
Résumé. - Les bandes d'absorption $v_{0-3}, v_{0-4}$ et $v_{0-5}$ des molécules isotopiques $\mathrm{H}{ }^{79} \mathrm{Br}$ et $\mathrm{H}{ }^{81 \mathrm{Br}}$ ont été analysées avec un spectromètre SISAM dont la résolution théorique est de l'ordre de $30 \mathrm{mK}$. Les mesures des nombres d'ondes des raies, effectuées avec une précision de $20 \mathrm{mK}$, ont permis de préciser la valeur des constantes de vibration et de rotation de chacune des molécules isotopiques.

Abstract. - The $v_{0-3}, v_{0-4}$ and $v_{0-5}$ bands of $\mathrm{H}{ }^{79} \mathrm{Br}$ and $\mathrm{H}{ }^{81} \mathrm{Br}$ were studied with a SISAM spectrometer having a theoretical resolving power of $30 \mathrm{mK}$. The wave number measurements of the vibration-rotation lines in the $v_{0-3}, v_{0-4}$ and $v_{0-5}$ bands have been obtained with a precision of $20 \mathrm{mK}$, and have allowed the determination of the molecular vibrational and rotational constants of each isotopic molecule.
\end{abstract}

1. Introduction. - Nous nous proposons de déterminer les valeurs des constantes de vibration et de rotation de l'acide bromhydrique gazeux par la mesure des nombres d'ondes des raies des bandes de vibrationrotation $v_{0-3}, v_{0-4}, v_{0-5}$. Nos prédécesseurs [1], [2], [3], [4], [5], [6] ne font pas mention des bandes $v_{0-3}$ et $v_{0-5}$. Il apparaît des désaccords entre les déterminations des constantes de vibration effectuées par Plyler [2] d'une part, par James [4] et Rank [5] d'autre part.

Nous avons donc entrepris l'étude des bandes $v_{0-3}$, $v_{0-4}$ et $v_{0-5}$ afin de lever l'indétermination qui subsiste sur les valeurs de ces constantes. Les bandes $v_{0-3}$ et $v_{0-5}$ n'avaient jamais été observées. Nous n'avons pas pu mettre en évidence la bande $v_{0-6}$ ni par enregistrement ni par photographie.

2. Appareillage et méthode expérimentale. - Nous avons utilisé le spectromètre de type SISAM déjà décrit [7], [8].

Le pouvoir de résolution théorique de l'appareil était de 240000 pour l'enregistrement de la bande $v_{0-3}$, de 300000 pour la bande $v_{0-4}$ et de 420000 pour la bande $v_{0-5}$. La source d'absorption est une cellule à réflexions multiples (longueur $1 \mathrm{~m}$ ) de type White. Les bandes ont été obtenues avec une longueur

(*) ERA n 303. d'absorption de 50 à $60 \mathrm{~m}$ et des pressions de $100 \mathrm{~mm}$ de $\mathrm{Hg}$ pour les bandes $v_{0-3}$ et $v_{0-4}$ et de $250 \mathrm{~mm}$ de

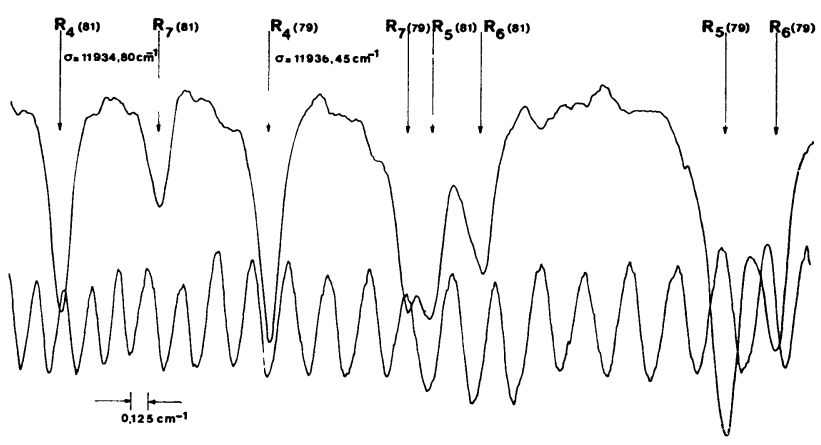

FIG. 1. - Retournement de la branche $\mathrm{R}$ de la bande $v_{0-5}$ de $\mathrm{HBr}$. Bande d'absorption $v_{0-5}$ de l'acide bromhydrique gazeux. Temps d'enregistrement $40 \mathrm{~min}$.

$\mathrm{Hg}$ pour la bande $v_{0-5}$. Un spectre cannelé en provenance d'un Fabry-Perot (épaisseur $2 \mathrm{~cm}$ ), des raies d'émission du thorium [9] et des gaz rares [10] ont permis l'étalonnage des spectres.

3. Résultat des mesures. - Nous avons pu mesurer pour chacune des bandes $v_{0-3}, v_{0-4}$ et $v_{0-5}$ une dizaine de raies $P$ et une dizaine de raies $R$. Les tableaux de ces mesures sont à la disposition des spécialistes intéressés auprès de la Rédaction du Journal de Physique. 
4. Constantes de l'état fondamental. - Pour calculer les constantes de l'état fondamental de vibration $\left(v^{\prime \prime}=0\right)$, nous avons formé la quantité $\Delta F^{\prime \prime}$ pour chaque bande et calculé le $\Delta F^{\prime \prime}$ moyen correspondant à l'ensemble des trois bandes.

Dans le tableau I sont réunies les valeurs de $\Delta F^{\prime \prime}(J)$ obtenues à partir de nos mesures et celles indiquées par Rank dans le cas de la molécule $\mathrm{H}^{81} \mathrm{Br}$. La comparaison de ces $\Delta F^{\prime \prime}(J)$ avec ceux de Rank montre l'excellente qualité de nos mesures.

\section{TABLEAU I}

$\begin{array}{ccc}J & \Delta F^{\prime \prime}\left(\mathrm{cm}^{-1}\right) & \Delta F^{\prime \prime} \text { (Rank) } \\ - & - & - \\ 1 & 50,079 & 50,079 \\ 2 & 83,439 & 83,438 \\ 3 & 116,762 & 116,757 \\ 4 & 150,019 & 150,011 \\ 5 & 183,217 & 183,200 \\ 6 & 216,313 & 216,294 \\ 7 & 249,278 & 249,282\end{array}$

Les valeurs des constantes du niveau fondamental de vibration, calculées par la méthode des moindres carrés sont rassemblées avec celles de Rank [5] dans le tableau II. Dans la limite de notre précision de mesure l'accord entre nos résultats et ceux de Rank d'une part et ceux de De Lucia, Helminger et Gordy [11] d'autre part, semble excellent.

5. Constantes des états excités. - Pour déterminer les constantes des états excités nous avons appliqué la méthode des moindres carrés aux quantités

$$
\mathrm{R}_{(J-1)}+\mathrm{P}_{(J)}
$$

pour chaque bande et chaque molécule isotopique.

Les variations de $D_{v}$ en fonction de $v$ sont extrêmement faibles pour l'acide bromhydrique et nous n'avons pu obtenir des résultats cohérents entre les diverses bandes étudiées.

En conséquence, nous avons déterminé les constantes $B_{v}$ et $v_{0 v}$, en considérant que $D_{v^{\prime \prime}}=D_{v^{\prime}}=D_{0}$. Cette approximation est valable dans la limite de la précision des résultats fournis (Tableau III).

\section{TABLEAU II}

\begin{tabular}{|c|c|c|c|c|}
\hline \multicolumn{3}{|c|}{$\mathrm{H}^{81 \mathrm{Br}}$} & \multicolumn{2}{|c|}{$\mathrm{H}{ }^{79} \mathrm{Br}$} \\
\hline & Calculé & Rank & Calculé & Rank \\
\hline & - & - & - & - \\
\hline$B_{0}\left(\mathrm{~cm}^{-1}\right)$ & $8,3487 \pm 0,0003$ & $8,348448 \pm 1 \times 10^{-5}$ & $8,3511 \pm 0,0003$ & $8,351030 \pm 1 \times 10^{-}$ \\
\hline$D_{0}$ & $3,45 \times 10^{-4} \pm 1 \times 10^{-5}$ & $3,4389 \times 10^{-4} \pm 1 \times 10^{-7}$ & $3,40 \times 10^{-4} \pm 1 \times 10^{-5}$ & $3,4410 \times 10^{-4} \pm 1 \times 10^{-}$ \\
\hline \multicolumn{5}{|c|}{ TABleAU III } \\
\hline$v^{\prime}$ & $B_{v^{\prime}}\left(\mathrm{cm}^{-1}\right)$ & $v_{0 v^{\prime}}\left(\mathrm{cm}^{-1}\right)$ & $B_{v^{\prime}}\left(\mathrm{cm}^{-1}\right)$ & $v_{0 v^{\prime}}\left(\mathrm{cm}^{-1}\right)$ \\
\hline - & - & - & - & - \\
\hline 3 & $7,6546 \pm 0,0005$ & $7404,181 \pm 0,005$ & $7,6566 \pm 0,0005$ & $7405,254 \pm 0,005$ \\
\hline 4 & $7,4223 \pm 0,0005$ & $9690,986 \pm 0,005$ & $7,4245 \pm 0,0005$ & $9692,351 \pm 0,005$ \\
\hline 5 & $7,1886 \pm 0,0005$ & $11886,291 \pm 0,005$ & $7,1907 \pm 0,0005$ & $11887,933 \pm 0,005$ \\
\hline
\end{tabular}

Les constantes moléculaires d'équilibre $B_{\mathrm{e}}, \alpha_{\mathrm{e}}, \gamma_{\mathrm{e}}$ et $\delta_{\mathrm{e}}$ ont été calculées en utilisant les coefficients $B_{4}$ et $B_{5}$ du tableau III et les valeurs de $B_{0}, B_{1}, B_{2}, B_{3}$ publiées par Rank [5]

$$
\begin{array}{r}
\mathrm{H}^{81} \mathrm{Br}\left\{\begin{array}{l}
B_{\mathrm{e}}=8,4650 \pm 2 \times 10^{-4} \mathrm{~cm}^{-1} \\
\alpha_{\mathrm{e}}=0,2336 \pm 5 \times 10^{-4} \mathrm{~cm}^{-1} \\
\gamma_{\mathrm{e}}=(10 \pm 3) \times 10^{-4} \mathrm{~cm}^{-1} \\
\delta_{\mathrm{e}}=(13 \pm 4) \times 10^{-5} \mathrm{~cm}^{-1}
\end{array}\right. \\
\mathrm{H}^{79} \mathrm{Br}\left\{\begin{array}{l}
B_{\mathrm{e}}=8,4677 \pm 2 \times 10^{-4} \mathrm{~cm}^{-1} \\
\alpha_{\mathrm{e}}=0,2337 \pm 5 \times 10^{-4} \mathrm{~cm}^{-1} \\
\gamma_{\mathrm{e}}=(10 \pm 3) \times 10^{-4} \mathrm{~cm}^{-1} \\
\delta_{\mathrm{e}}=(13 \pm 4) \times 10^{-5} \mathrm{~cm}^{-1}
\end{array}\right.
\end{array}
$$

Le calcul des constantes de vibration s'effectue d'une manière analogue.

Une bonne représentation du spectre nécessite la présence d'un terme du quatrième degré en $v$. Afin d'augmenter la précision du résultat, nous avons introduit les centres des bandes mesurés par Rank et ceux que nous avons déterminés, et traité dans un même calcul le cas de la molécule $\mathrm{H}^{81} \mathrm{Br}$ et celui de $\mathrm{H}^{79} \mathrm{Br}$, puisque le rapport des masses réduites $\rho^{2}$ est connu avec une grande précision. Dans ces conditions les constantes calculées diffèrent notablement de celles publiées à ce jour

$$
\begin{gathered}
\mathrm{H}^{81} \mathrm{Br}\left\{\begin{array}{rr}
\omega_{\mathrm{e}}=2 & 649,308 \pm 1 \times 10^{-2} \mathrm{~cm}^{-1} \\
\omega_{\mathrm{e}} x_{\mathrm{e}}= & 45,516 \pm 1 \times 10^{-2} \mathrm{~cm}^{-1} \\
\omega_{\mathrm{e}} y_{\mathrm{e}}= & 0,107 \pm 5 \times 10^{-3} \mathrm{~cm}^{-1} \\
\omega_{\mathrm{e}} z_{\mathrm{e}}= & 0,0135 \pm 5 \times 10^{-4} \mathrm{~cm}^{-1}
\end{array}\right. \\
\mathrm{H}^{79} \mathrm{Br}\left\{\begin{array}{rr}
\omega_{\mathrm{e}}=2 & 649,721 \pm 1 \times 10^{-2} \mathrm{~cm}^{-1} \\
\omega_{\mathrm{e}} x_{\mathrm{e}}= & 45,530 \pm 1 \times 10^{-2} \mathrm{~cm}^{-1} \\
\omega_{\mathrm{e}} y_{\mathrm{e}}= & 0,107 \pm 5 \times 10^{-3} \mathrm{~cm}^{-1} \\
\omega_{\mathrm{e}} z_{\mathrm{e}}= & 0,0135 \pm 5 \times 10^{-4} \mathrm{~cm}^{-1} .
\end{array}\right.
\end{gathered}
$$

6. Conclusion. - Ce travail a permis la détermination des constantes rotationnelles et vibrationnelles de l'acide bromhydrique gazeux. Il subsiste une imprécision sur la constante de distorsion centrituge $D_{v}$. Il est possible de comparer nos résultats à ceux obtenus 
par Rank et ses collaborateurs [5]. Les constantes de rotation que nous annonçons sont compatibles avec les leurs, mais moins précises parce que nous n'avons obtenu que des raies correspondant à des valeurs de $J$ faibles avec une précision effective de pointé dix fois moins bonne. Pourtant la mesure des bandes nouvelles harmoniques élevées a permis de préciser la valeur des constantes moléculaires de vibration. L'étude de ces bandes de faible intensité a été possible grâce à la grande clarté et à la haute résolution du spectromètre, qui permettent de conserver un rapport signal sur bruit compatible avec la précision des mesures même lorsque le nombre de réflexions dans la cuve devient important.

Malheureusement, la précision des mesures n'est pas tout à fait celle que laisse espérer le pouvoir de résolution, en grande partie à cause des défauts mécaniques qui interviennent pendant la rotation des réseaux.

Remerciements. - Nous ne voulons pas terminer la présentation de ce travail sans exprimer notre très vive gratitude à $M$. le Professeur Schiltz. Nous remercions également $\mathbf{M}$. Gonzales qui a mis au point les programmes utilisés dans cette étude.

\section{Bibliographie}

[1] Naude, S. M. et Verleger, H., Proc. Phys. Soc. A 63 (1950) 470.

[2] Plyler, E. K., J. Res. Nat. Bur. Stand. (U. S.) 63 (1960) 377.

[3] Mould, H. M., Price, W. C. et Wilkinson, G. R., Spectrochim. Acta 16 (1960) 479.

[4] James, T. C. et Thibault, R. J., J. Chem. Phys. 42 (1965) 1450.

[5] Rank, D. H., Fink, U., Wiggins, T. A., J. Mol. Spect. 18 (1965) 170-183.

[6] Jones, G. E., et Gordy, W., Phys. Rev. 136 (1964) A 1229.
[7] Bernage, P., Houdart, R., Niay, P., Spectrochim. Acta 26B (1971) 261-270.

[8] Bernage, P., Niay, P., Bocquet, H., Houdart, R. et CARLIER, P., Nouv. Revue Opt. Appl. 3 (1972) 89-93.

[9] Connes, P., Guelachvili, G., Maillard, J. P., Michel, G. et Connes, J., Delouis, H., Nouv. Revue Opt. Appl. 1 (1970) 3-22.

[10] Rao, K. N., HumphreYs, C. S., RANK, D. H., Wavelength standards in the infrared (Academic press), 1966.

[11] De Lucia, F. C., Helminger, P., Gordy, W., Phys. Rev. A 3 (1971) 1849. 